\title{
Chronic lymphocytic leukemia: A tale of one or two signals?
}

Cell Research (2013) 23:182-185. doi:10.1038/cr.2012.152; published online 13 November 2012

The significant correlation between disease aggressiveness and the gene and protein structures of the B-cell receptors (BCRs) expressed on chronic lymphocytic leukemia (CLL) cells, together with the evidence for chronic activation of the BCR pathway, have led to the hypothesis that this leukemia initiates and progresses by selecting normal $B$ lymphocytes reactive with a restricted set of (auto)antigens. A study recently published in Nature identified a novel signal-initiating interaction between the third complementary determining region of the IG heavy chain variable domain (HCDR3) and an epitope in the second framework region (FR2) that appears to be unique to CLL B cells and that calls into question the need for classical antigen binding in the activation and expansion of the leukemic cells. These findings are discussed in the context of available information about the antigen reactivity of CLL B cells and its potential role in clonal survival and drive.

Surface membrane immunoglobulin (smIg), the antigen recognition unit of the BCR, is clonally restricted in structure and is essential for the development, survival, and growth of $\mathrm{B}$ cells. The smIgs of CLL B cells exhibit distinct structural features $[1,2]$. They frequently use $I G H V$ and $I G L V \kappa / \lambda$ genes differing from those of B lymphocytes in normal individuals and, not infrequently, associate specific $I G H V$ s with specific $I G H D-J \mathrm{~s}$ and specific $I G L V \mathrm{~s}$ with specific $I G L J_{\mathrm{s}}$, leading to remarkably similar "stereotyped" HCDR3s and
smIgs. Moreover, CLL IGHVs differ in the presence or absence of somatic mutations, which correlates with dramatically divergent patient outcomes: $I G H V$ unmutated (U-CLL) patients experience more aggressive clinical courses than $I G H V$ mutated (M-CLL) patients. Lastly, these structural differences group CLL BCRs into 3 categories: those with sets of stereotyped smIgs ( $33 \%$ of patients) that are more likely U-CLLs, those with smIgs using specific $I G H V \mathrm{~s}$ roughly equally distributed between U-CLL and M-CLL, and those without obvious $I G V$ characteristics that are more frequently M-CLLs [1, 2].

These and related findings led to the hypothesis that selection and expansion of B lymphocytes with specific BCR structures is integral for the development and probably evolution of CLL [1]. Furthermore, it is thought that these B lymphocytes are culled from either a restricted B-cell subset/lineage or the B-cell pool at large by binding restricted (classes of) foreign or autoantigens, and therefore that signaling through BCRs, mediated by binding these ligands, is responsible for the survival and expansion of the selected B cells.

Regarding antigen selection, because stereotyped BCRs can be found in unrelated patients from different parts of the world and most CLL clones with stereotyped BCRs lack significant numbers of $I G H V$ mutations (U-CLL), the selecting ligands in these cases have been considered to be autoantigens. In contrast, the less structurally restricted M-CLL smIgs have been envisioned as subject to foreign antigen selection.
This (auto)antigenic epitope reactivity difference has been borne out, in general, by studies of soluble CLL Igs indicating frequent poly(auto)reactivity for U-CLL-derived Igs and less so for M-CLL Igs [3-5]. Since conversion of some M-CLL $I G H V$ s to the germline sequence restores autoreactivity, common derivation of both CLL subtypes from autoreactive $\mathrm{B}$ cells has been put forward [6].

As for BCR signaling, considerable evidence exists for ongoing stimulation of CLL cells in vivo, including phosphorylation of signaling kinases and upregulation of associated transcription factors in unmanipulated CLL cells, which, when inhibited or downregulated, lead to apoptosis [7-9]. The extent that these signals are tonic or induced by antigen binding is not clear, although data support both possibilities.

Thus, the prevailing view is that CLL develops from B lymphocytes bearing smIgs that can be biased in structure, chosen from the B-lymphocyte repertoire by (auto)antigen selection, and having received repetitive BCR-mediated signals that promote development of requisite DNA or functional abnormalities to drive clonal expansion and ultimate transformation. This process of tumor development is influenced by the tissue microenvironment that provides supportive receptor-ligand interactions (BCR-antigen and other receptors, e.g., for cytokines and chemokines).

Recently, Dühren-von Minden et al. [10] reported that inserting CLL smIgs into a murine pre-B-cell line lacking expression of the pre-BCR and BCR, 
and transfected with an inducible BCR signaling adaptor molecule (SLP65/ BLNK), led to intracellular signaling/ $\mathrm{Ca}^{++}$flux in the apparent absence of ligands interactive with the smIgs. This signaling did not occur when Igs from four different non-Hodgkins lymphoma cells were transferred in a like manner. Remarkably, signaling was observed with all CLL smIgs tested, regardless of the type transferred, i.e., stereotyped, $I G H V$-restricted, or $I G V$-non-descript, and also regardless of $I G H V$ mutation status. This principle also held for smIgs derived from $\mathrm{CD}^{+} \mathrm{B}$-cell clones emerging spontaneously in a murine CLL-like model (TCL1 transgenic mice), but not in $\mathrm{CD}^{+} \mathrm{B}-1 \mathrm{a} \mathrm{B}$ cells from normal, non-TCL1 animals. The important interaction unit within the smIgs of these BCRs resided in the HCDR3s, because insertion of CLL HCDR3s converted a normal human smIg that did not signal into an autonomously active receptor.

Expanding on peptide-phage display studies by others identifying an amino acid sequence that bound human Igs [11], the authors found a related sequence in the FR2 of human $I G H V \mathrm{~s}$ that appears to interact with the HCDR3s of CLL smIgs; abrogating $\mathrm{Ca}^{++}$flux by changing specific, single amino acids of the "internal epitope" supported this. Of note, signaling did not require other cells, as single cells exhibited $\mathrm{Ca}^{++}$ flux if a CLL BCR was in place. Thus, the authors conclude that CLL cells are driven by an antigen-independent, cell-autonomous signaling mechanism that is inherent to the structure of the leukemic BCRs, involving an HCDR3internal epitope interaction.

These are novel and provocative findings that corroborate the importance of BCR structure in the genesis and progression of CLL. Moreover, like many important findings, they challenge prevailing beliefs, in particular how the BCR influences CLL cell biology, thereby creating new sets of possibilities and questions.

The findings identify a new process whereby CLL HCDR3s interact with epitopes within the same or adjacent BCRs. However, we suggest that this is not antigen independent. In fact, this type of reactivity could be viewed as exemplary autoreactivity, with exquisite selection of $\mathrm{B}$ cells with smIgs containing HCDR3s that recognize FR2 autoepitopes. Other examples of autonomous or inter-cell reactivities of this type, albeit less well-defined at the epitope level, would be anti-Fab- or anti-IgG heavy chain-reactive $B$ cells seen in normal and autoimmune settings. As a class, these may be considered BCRsuperantigen interactions.

Key unanswered questions involve the functional consequences of this type of BCR engagement for a CLL B cell, the role for engagement of the same BCR with other "external" epitopes on non-smIg antigens, and the anatomic context in which these two types of interactions could occur.

Several parameters influence the ability of BCR-antigen engagement to generate a signal, e.g., interaction affinity and duration and antigen valency, and these are in turn shaped by concurrent inputs by other cells. The importance of duration and valency of CLL cell triggering has been documented using solid phase anti-IgM antibodies as surrogate antigens (Figure 1A) [12]. Although the affinity of the HCDR3-internal epitope interaction is yet to be measured, this is probably single epitope binding and therefore may not be high. Also, the strength and duration of binding may be affected by intra- and inter-molecular competition for HCDR3-FR2 interactions: HCDR3 interacting with the FR2 on the same smIg molecule, which is not likely to lead to effective signaling because of a lack of BCR crosslinking (Figure 1C), and HCDR3 interacting with the FR2 of other smIgs (Figure 1B), which would lead to BCR crosslinking. Furthermore, competition for HCDR 3 and other CDRs by epitopes on conventional auto- and foreign antigens needs to be considered. Although the authors have not found such a competition between anti-hapten reactivity and anti-internal epitope interactions for a specific anti-NIP smIg, it is possible that other smIgs with different specificities could be affected by such a process. Collectively, these factors could diminish the "strength" of the BCR signal delivered by internal epitope binding, consistent with the observation that SYK, which is one of the most BCRproximal signaling molecules, is only partially activated in unmanipulated CLL cells [13].

Moreover, basal $\mathrm{Ca}^{++}$levels are elevated in unmanipulated CLL B cells, and this has been interpreted as indicative of a state of anergy due to ongoing activation in vivo by (auto) antigen binding. Elevated levels of $\mathrm{Ca}^{++}$ could not be detected in the cell lines used in this study because signaling could not occur until SLP65/BLNK levels were induced. Hence, it would be interesting to know whether $\mathrm{Ca}^{++}$ levels remain elevated in cells bearing CLL BCRs after SLP65/BLNK induction; second, whether these cells can be subsequently triggered by smIgM crosslinking; and third, whether the duration of $\mathrm{Ca}^{++}$elevation and responsiveness to smIgM stimulation differs between cells expressing U-CLL and M-CLL BCRs. This information would help associate the in vitro analyses reported by the authors with the biology of CLL cells in vivo, in particular whether the HCDR3-internal epitope signaling process is responsible for the "anergic signaling phenotype". Although anergy is thought to correlate with shortened cell survival, for murine B-1 cells, a possible precursor of human CLL cells, chronic signaling, leading to a "tolerant" phenotype, is associated with prolonged survival [14]. Hence, the basal $\mathrm{Ca}^{++}$ signaling of CLL cells might lead to enhanced survival without an accompanying expansion; the induction of apoptosis in CLL cells by $\mathrm{Ca}^{++}$channel blockers and cyclosporine A may reflect this [15]. Thus, HCDR3-internal epitope 


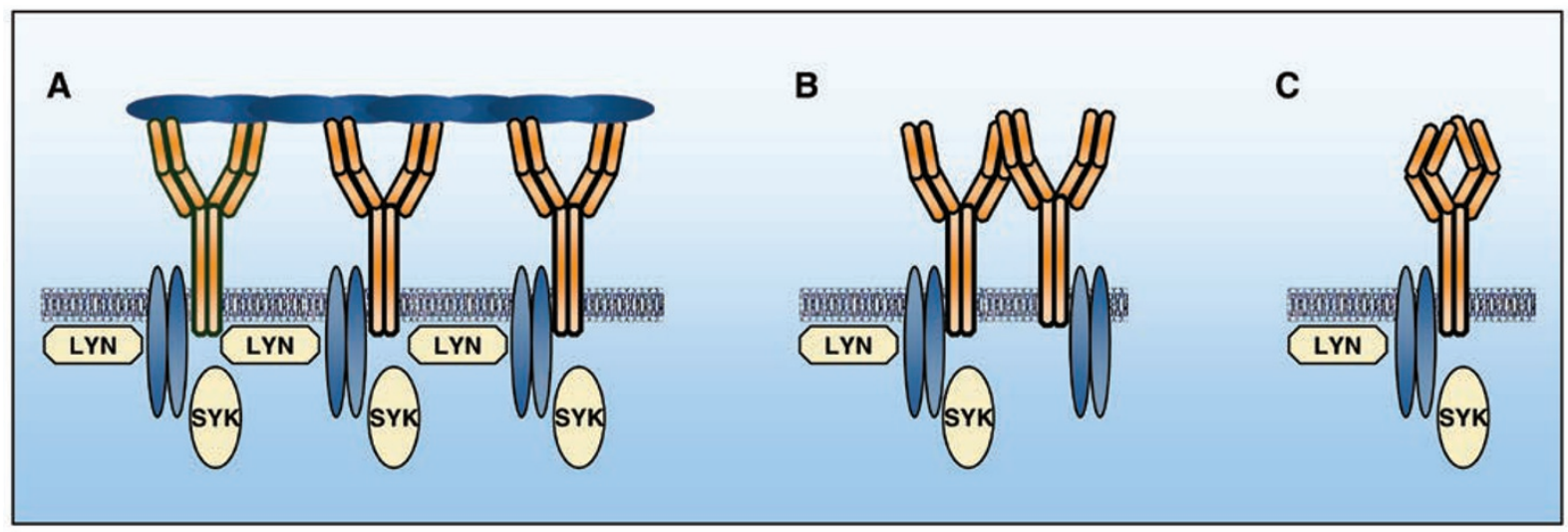

Figure $1 \mathrm{BCR}$ signals generated in CLL B cells. (A) Conventional antigen-dependent BCR signal generated by crosslinking of neighboring BCRs by an external self- or foreign antigen or an antigen surrogate such as anti-lgM antibodies. (B) Cell-autonomous BCR signal generated by an inter-molecular interaction between the HCDR3 of one smlg and the FR2 of a neighboring smlg. (C) Cell-autonomous BCR signal generated by an intra-molecular interaction between the HCDR3 and FR2 of the same smlg.

signaling may not necessarily "drive" CLL cells but allow them to survive longer to eventually receive other expansion signals, consistent with internal epitope-mediated signaling playing an early role in promoting the growth of the precursors of CLL, which in some cases has been identified years before diagnosing the disease [16].

M-CLL and U-CLL cells differ in their abilities to transmit anti-IgMmediated BCR signals and to bind autoantigens, with the former doing both poorly and the latter more effectively. These differences could best fit a scenario in which basal HCDR3-internal epitope signals are generated in both M-CLL and U-CLL cells, leading to their prolonged survival, whereas signals initiated by "typical" (auto)antigen binding are generated primarily in U-CLL cells, leading to their proliferation and subsequent clonal expansion. In support of this possibility, it should be noted that internal epitope signaling did not distinguish between U-CLL patients with more aggressive clinical courses and M-CLL patients with more benign outcomes, suggesting that additional factors must be important in determining the course of the disease. In this regard, CLL cells depend on interactions with non-leukemic cells in lymphoid tissues for survival and expansion signals, and it appears that expansion signals are delivered at least in part through the BCR. Solid tissues can provide stimulatory antigens, including autoantigens generated during apoptosis, in an immobilized state leading to BCR engagement of longer duration, increasing the likelihood that standard BCR-antigen interactions would take place. Such interactions could also provide the co-stimulatory signals that are required for leukemic cell proliferation. It is not clear that cells undergoing cellautonomous stimulation would need to re-enter solid lymphoid tissues, unless BCR-internal epitope signaling also requires trophic influences from cells of the microenvironment for CLL cell survival and expansion.

The study by Dühren-von Minden et al. [10] is fascinating, novel, and possibly paradigm-setting, especially if this feature is a requisite for leukemogenesis. The findings open a new dimension for CLL cell biology and require re-thinking some existing premises. We suggest, however, based on available information about CLL and normal B lymphocytes and not experimental evidence, that the interpretations of these findings are not straightforward and will require targeted studies, in particular relating to the functional consequences, such as survival and expansion, to define the individual roles of internal epitope and classical (auto)antigen epitope binding in the pathogenesis of CLL.

\section{Nicholas Chiorazzi ${ }^{1}$, Dimitar G Efremov ${ }^{2}$}

${ }^{1}$ Karches Center for Chronic Lymphocytic Leukemia Research, The Feinstein Institute for Medical Research, Hofstra North Shore-LIJ School of Medicine, 350 Community Drive, Manhasset, NY 10030, USA; ${ }^{2}$ Molecular Hematology, International Centre for Genetic Engineering and Biotechnology, CNR Campus "A. BuzzatiTraverso", Rome, Italy

Correspondence: Nicholas Chiorazzia, Dimitar G Efremov ${ }^{\mathrm{b}}$

aE-mail: nchizzi@NSHS.edu

bE-mail: efremov@icgeb.org

\section{References}

1 Chiorazzi N, Rai KR, Ferrarini M. $N$ Engl J Med 2005; 352:804-815.

2 Agathangelidis A, Darzentas N, Hadzidimitriou A, et al. Blood 2012; 119:4467-4475.

3 Lanemo Myhrinder A, Hellqvist E, Sidorova E, et al. Blood 2008; 111:3838-3848.

4 Catera R, Silverman GJ, Hatzi K, et al. Mol Med 2008; 14:665-674.

5 Chu CC, Catera R, Zhang L, et al. Blood 2012; 115:3907-3915.

6 Hervé M, Xu K, Ng YS, et al. J Clin Invest 2005; 115:1636-1643.

7 Muzio M, Apollonio B, Scielzo C, et al. Blood 2008; 112:188-195.

8 Gobessi S, Laurenti L, Longo PG, et al. 
Leukemia 2009; 23:686-697.

9 Herishanu Y, Pérez-Galán P, Liu D, et al. Blood 2011; 117:563-574.

10 Dühren-von Minden M, Übelhart R, Schneider D, et al. Nature 2012; 489:309-312.

11 Binder M, Müller F, Jackst A, et al. Cancer
2011; 117:1891-1900.

12 Petlickovski A, Laurenti L, Li X, et al. Blood 2005; 105:4820-4827.

13 Carsetti L, Laurenti L, Gobessi S, et al. Cell Signal 2009; 21:1187-1194.

14 Chumley MJ, Dal Porto JM, Kawaguchi S, et al. J Immunol 2000; 164:4586-4593.

15 Vilpo J, Koski T, Vilpo L. Haematologica 2000; 85:806-813.

16 Landgren O, Albitar M, Ma W, et al. $N$ Engl J Med 2009; 360:659-667. 